\title{
Recognizing Digital Biomarkers for Fatigue Assessment in Patients with Multiple Sclerosis
}

\author{
Liliana Barrios \\ Department of Computer Science \\ ETH Zurich \\ Zurich, Switzerland \\ liliana.barrios@inf.ethz.ch \\ Silvia Santini \\ Faculty of Informatics \\ Universita della Svizzera Italiana \\ Lugano, Switzerland \\ silvia.santini@usi.ch
}

\author{
Pietro Oldrati \\ Department of Computer Science \\ ETH Zurich \\ Zurich, Switzerland \\ oldratip@ethz.ch \\ Andreas Lutterotti \\ Department of Neurology \\ University of Zurich \\ Zurich, Switzerland \\ andreas.lutterotti@usz.ch
}

\begin{abstract}
We describe our ongoing work on the design and implementation of a system to continuously monitor different physiological parameters in patients with multiple sclerosis (MS). We focus specifically on monitoring functions of the autonomous nervous system and activities of daily life using wearable and mobile sensors and to correlate these with important symptoms of MS, in particular, fatigue. Fatigue is a highly prevalent and debilitating symptom in MS patients. However, the underlying cause and pathogenetic mechanisms are poorly understood and consequently therapeutic interventions limited. As the first step in our research effort, we evaluate the feasibility of off-the-shelf devices to record several physiological parameters in MS patients continuously.
\end{abstract}

\section{KEYWORDS}

Mobile health; Multiple Sclerosis; Fatigue assessment using physiological sensors.

\section{INTRODUCTION}

The recent advances in mobile and wearable technologies opened up opportunities to improve healthcare monitoring systems. Using devices like smartphones and smartwatches it is possible to collect sensor data continuously, e.g., mobility or physiological data, that can be linked to the health status of the owner of the devices. This is particularly promising for the management of chronic conditions, where the monitoring of patients' physiological parameters over extended periods of time could lead to a better understanding of diseases and improvement of therapies. Previous studies already explored the use of mobile and wearable technologies to monitor patients with diabetes [8], parkinson [9], or hypertension [7]. However, other complex diseases such as multiple sclerosis have received less attention.

Multiple Sclerosis (MS) is a chronic inflammatory disease of the central nervous system characterized by recurrent demyelinating lesions in the brain and spinal cord, leading to nerves damage and ultimately neurodegeneration. With more than two million patients worldwide, MS is one of the leading causes of neurological disability in young adults $[4,10]$. MS patients are affected by a wide variability of symptoms, including fatigue, sensory problems, difficulties in motor function and coordination, cognitive deficits and bladder disorders. The autonomous nervous system is frequently affected in MS patients, but its impact on different symptoms, like fatigue, is only poorly understood. A detailed assessment of the autonomous nervous system in the standard clinical environment is challenging and hampered by short-term or even single time-point measurements. Multi-sensor wearable devices overcome these limitations by allowing unobtrusive continuous monitoring over long-term periods. With their light-weight and small size characteristics, they enable to assess patients in their daily environment. Additionally, these devices are less costly than medical-grade monitoring systems, making them more accessible. Moreover, such a system could be as well beneficial for patients in countries or locations where access to expensive medical monitoring systems is limited.

The goal of our work is to leverage mobile and wearable devices to enable continuous monitoring of functions of the autonomic nervous system in MS and allow patients to report their symptoms and perform medical tests independently and on a regular basis, i.e., weekly or even daily. In this context, we currently focus on measuring autonomous nervous system functions continuously and correlate it with important symptoms, in particular, fatigue. Fatigue has a significant impact on the quality of life of patients, as it affects work performance as well as personal interactions. Despite being highly prevalent and being a debilitating symptom, it is not well understood, and there are no objective parameters to diagnose and quantify the level of fatigue. Researchers have highlighted the need for a better understanding of the underlying causes of fatigue and the implementation of new therapeutic strategies [12]. We 


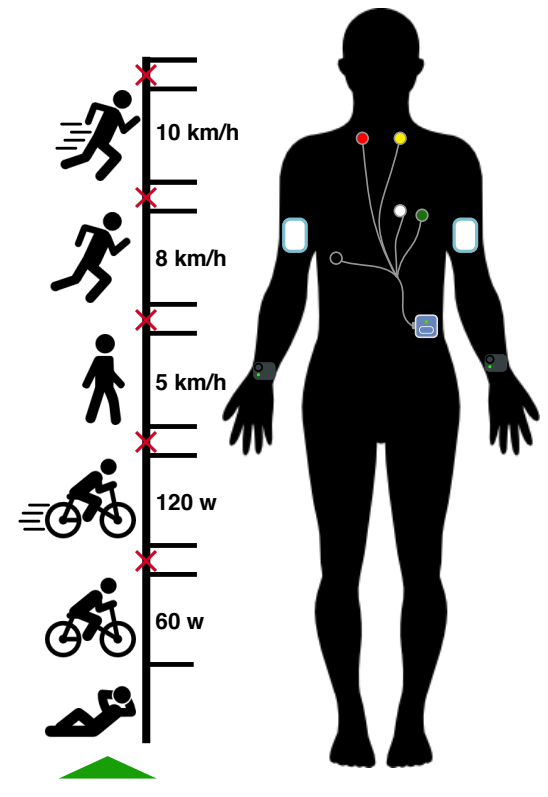

Figure 1: Sensor validation protocol at the left side and sensor placement at the right side. Empatica E4 devices placed on the subject's wrist; Everion devices on the arms; and the Holter monitor attached with five electrodes to the chest.

plan to use wearable sensors to measure physiological parameters - including electrodermal activity and cardiovascular parameters of MS patients continuously and over extended periods of time. By letting patients use their smartphones to report perceived fatigue levels, we expect to collect sufficient evidence to define a datadriven model of fatigue. This will, in turn, allow us to develop a system to unobtrusively and continuously measure fatigue using wearable sensors only.

In this work, we present preliminary results in validating the feasibility of off-the-shelf sensors, i.e., the Everion [1] and Empatica E4 [2] devices, to be used to assess functions of the autonomous nervous system. Our results show that the data provided by the two devices under test is in good agreement with the signals supplied by a medical-grade Holter monitor.

\section{SENSOR VALIDATION: PRELIMINARY RESULTS}

Using wearable sensors, we aim to correlate measurements of daily activities and autonomic nervous system function with the frequency and severity of fatigue in MS patients. Our goal is to examine the role of the autonomic nervous system in MS-associated fatigue and to develop new objective measures to follow fatigue in MS patients.

As the first step in this direction, we considered two off-the-shelf sensors capable of measuring heart rate through photoplethysmography (PPG) and electrodermal activity (EDA): the Empatica E4 [2] and the Everion [1] devices. For assessing the validity of the PPG sensors, we use a simplified protocol similar to the one followed in [5]. The protocol initiates with 5 minutes of resting period
Table 1: Values of features commonly used in the analysis of heart rate (beats per minute) signals: Mean, standard deviation (STD), and Root Mean Square of the Successive Differences (RMSSD). In particular, the features computed from the data collected by the Everion device show a very good agreement with the corresponding features of the medicalgrade Holter monitor.

\begin{tabular}{c|ccc}
\hline Device & Mean & STD & RMSSD \\
\hline Holter Monitor & 128.5 & 27.8 & 20 \\
Everion Right & 122.8 & 26.5 & 24.3 \\
Empatica Right & 111.2 & 23.9 & 67.7 \\
\hline
\end{tabular}

followed by five activities: biking (60W), biking (120W), walking $(5 \mathrm{~km} / \mathrm{h})$, jogging $(8 \mathrm{~km} / \mathrm{h})$ and running $(10 \mathrm{~km} / \mathrm{h})$. Each activity lasts for 5 minutes, and between each activity, there is a resting period of 5 minutes. The left side of Figure 1 depicts our validation protocol.

We conducted a preliminary test with one participant wearing two Empatica E4 devices (one on each wrist), two Everion devices (one per arm) and a medical-grade Holter monitor from General Electric [3] with 5 electrodes attached to the body. The sensor placement is depicted on the right side of Figure 1. Figure 2 shows the signals collected from all three devices. The signals show a good agreement, with the data from the Everion being almost identical to the one of the Holter monitor. Table 1 shows the values of common features used to analyze ECG signals. The values for all three devices are very similar, whereas the Everion shows the best agreement with the Holter monitor.

\section{RELATED WORK}

Yu et al. [13] created a portable wireless system that can differentiate between fatigued MS patients and matched healthy controls. Results from this work show that MS patients have alternations of the heart rate $(\mathrm{HR})$ and $\mathrm{HR}$ frequency depending if the test required cognitive or physical effort. Similarly, we aim at measuring physiological data and possibly identify alteration of the autonomic nervous system in MS patients. However, instead of combining multiple sensors to build a measuring system, we take advantage of the advances in wearable technologies and use off-the-shelf devices.

Kim et al. [6] developed a real-time digital fatigue score (RDFS) by using watches programmed to beep four times a day to query patients about there fatigue level. We propose using a similar approach for real-time assessment of fatigue using smartphones.

The work by Sehle et al. [11] aims to quantify MS-related fatigue by using kinematic gait analysis objectively. They found correlation between physical measurements and subjective fatigue scales. In our work, we consider sensors with integrated accelerometers to estimate physical activity.

In summary, previous research on fatigue has focused on using dedicated sensors or devices to investigate the symptom. In some cases, only one sensor or device was considered $[6,11]$. In other instances [13], multiple sensors were employed simultaneously to develop a measuring system. However, only short-time assessments were conducted. We build upon this basis and the advances of 


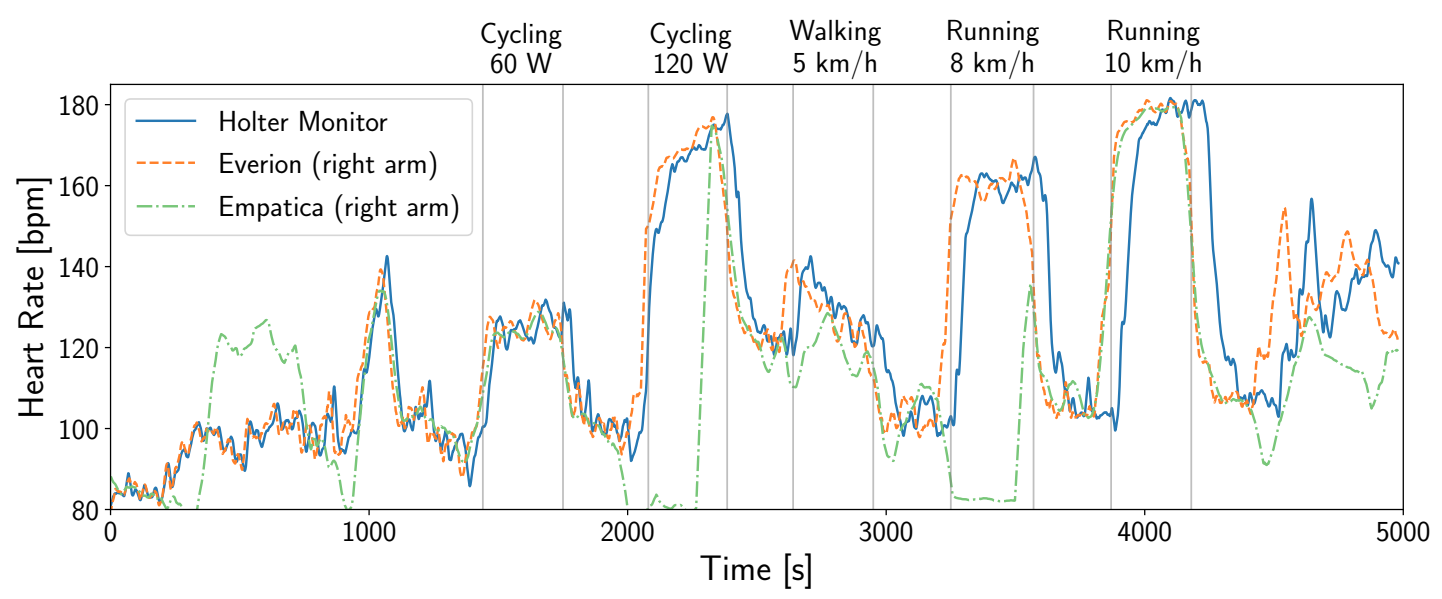

Figure 2: Signals collected using the two devices under test and a medical-grade Holter monitor. In particular, the Everion device by Biovotion shows a very good agreement with the data of the Holter monitor.

technology to propose a system based on a single off-the-shelf multisensor wearable device and a dedicated smartphone application. Wearable devices are light-weight and small size making them easy to handle and allow for unobtrusive continuous monitoring over a long-term period of time.

\section{CONCLUSIONS AND FUTURE WORK}

We report preliminary test results evaluating the feasibility of offthe-shelf devices to be used to monitor fatigue in patients with MS Our tests show that the signals measured by both devices under test are in good agreement with the data recorded by a medicalgrade device, whereas the Everion monitor is the one with the best agreement. As part of our future work, we plan to run more tests with several participants and to analyze the obtained data in detail.

\section{REFERENCES}

[1] Biovotion AG 2018. Everion monitor. Retrieved April 02, 2018 from http: //www.biovotion.com

[2] Empatica Inc. 2018. E4 wristband. Retrieved April 02, 2018 from https://www. empatica.com/en-eu/research/e4/

[3] General Electric Healthcare 2018. Holter Recorder SEER* 1000. Retrieved April 02, 2018 from http://www3.gehealthcare.com/en/products/categories/diagnostic ecg/ambulatory/seer_1000

[4] Stephen L. Hauser and Jorge R. Oksenberg. 2006. The Neurobiology of Multiple Sclerosis: Genes, Inflammation, and Neurodegeneration. Neuron 52, 1 (2006), 61 76.

[5] Edward Jo, Kiana Lewis, Dean Directo, Michael J Kim, and Brett A Dolezal. 2016 Validation of Biofeedback Wearables for Photoplethysmographic Heart Rate Tracking. F Sports Sci Med 15, 3 (2016), 540-547.

[6] Edward Kim, Jesus Lovera, Laura Schaben, J. Melara, Dennis Bourdette, and Ruth Whitham. 2010. Novel method for measurement of fatigue in multiple sclerosis Real-Time Digital Fatigue Score. 7 Rehabil Res Dev 47, 5 (2010), 477-84.

[7] K. Kusk, D. B. Nielsen, T. Thylstrup, N. H. Rasmussen, J. Jørvang, C. F. Pedersen, and S. Wagner. 2013. Feasibility of using a lightweight context-aware system for facilitating reliable home blood pressure self-measurements, In PervasiveHealth PervasiveHealth, 236-239.

[8] H Lee, T Choi, Y Lee, H Cho, R Ghaffari, L Wang, H Choi, T Chung, N Lu, T Hyeon, S Choi, and D Kim. 2016. A graphene-based electrochemical device with thermoresponsive microneedles for diabetes monitoring and therapy. Nat Nanotechnol 11 (2016), 566 EP -.

[9] Sinziana Mazilu, Ulf Blanke, Michael Hardegger, Gerhard Tröster, Eran Gazit, and Jeffrey M. Hausdorff. 2014. GaitAssist: A Daily-life Support and Training System for Parkinson's Disease Patients with Freezing of Gait. In Proc. CHI. ACM Press, 2531-2540.
[10] National Multiple Sclerosis Society 2018. Who Gets MS? (Epidemiology). Retrieved April 30, 2018 from https://www.nationalmssociety.org/What-is-MS/ Who-Gets-MS

[11] Aida Sehle, Annegret Mündermann, Klaus Starrost, Simon Sailer, Inna Becher, Christian Dettmers, and Manfred Vieten. 2011. Objective assessment of motor fatigue in multiple sclerosis using kinematic gait analysis: a pilot study. 7 Neuroeng Rehabil 8 (2011), 59.

[12] Ayache SS and Chalah MA. 2017. Fatigue in multiple sclerosis - Insights into evaluation and management. Neurophysiol Clin 47 (2017), 139-171.

[13] Fei Yu, Arne Bilberg, Ulrik Dalgas, and Egon Stenager. 2013. Fatigued patients with multiple sclerosis can be discriminated from healthy controls by the recordings of a newly developed measurement system (FAMOS): a pilot study. Disabil Rehabil Assist Technol 8, 1 (2013), 77-83. 